\title{
Macrolide Antibiotic in Opioid Detoxification: A Serendipitous discovery?
}

\author{
Aich TK ${ }^{1}$, Haider $\mathbf{J}^{2}$, Dhungana $\mathbf{M}^{3}$ \\ 1. Professor \& Head, Department of Psychiatry I/C OST Centre, BRD Medical College, Gorakhpur, UP, \\ India, 2. Associate Professor \& Head, Department of Pharmacology, BRD Medical College, Gorakhpur, \\ UP, India, 3. Lecturer, Department of Psychiatry, Devdaha Medical College and Research Institute, \\ Rupandehi, Nepal
}

E-mail *Corresponding author : jamalatbrd@gmail.com

\begin{abstract}
Introduction: While receiving opioid detoxification treatment with standard Detoxification protocol, one patient was additionally prescribed Roxithromycin for his associated skin infection. Upon withdrawal of roxithromycin following improvement in skin infection, very next day same patient complained of heightened opioid withdrawal symptoms. These symptoms relieved again following reintroduction of Roxithromycin!

Material And Method: It was a naturalistic study of 72 Opioid Dependent patients who received inpatient detoxification treatment at the Universal College of Medical Sciences, Bhairahawa, Nepal. A total of 16 patients received standard opioid detoxification protocol before the incidence of above mentioned patient. Subsequently, a total of 56 patients received Roxithromycin, in addition to the standard protocol, during their inpatient stay with a maximum for a period of 10 days. Necessary ethical clearance from the institute's ethical clearance committee was taken before carrying out this trial. Duration of inpatient stay and follow-up pattern after discharge was taken as the objective assessment of the efficacy of Roxithromycin in reducing severity of withdrawal symptoms. All other treatment parameters were similar in two groups.

Results: Two groups did not differ in most demographic and clinical variables compared. Opioid dependence patients who received Roxithromycin had significantly longer $(t=2.5 ; p=0.01)$ voluntary hospitalization stay $(10.6$ days, $S D=6.2)$ vis-à-vis patients who did not receive it $(6.4$ days, $S D=3.9)$. They also reported significantly more number of follow-ups after discharge (Fisher's Exact Test $=0.02$ ).

Conclusion: Roxithromycin, besides being an antibiotic, also possibly act as an anti-inflammatory and immunemodulatory agent by regulating leukocyte function
\end{abstract}

Keywords: Opioid Detoxification, Roxithromycin, Anti-Inflammatory Action, Treatment Of Withdrawal

\section{INTRODUCTION}

The 'Opioid Detoxification' process include use of opioid agonists (e.g., methadone), partial agonists (e.g., buprenorphine), antagonists (e.g., naloxone, naltrexone), or non-opioid alternatives such as clonidine, benzodiazepines, and nonsteroidal anti-inflammatory agents. In many cases, one or more medications are combined.1-4 The choice of detoxification medication and the duration of the process depend on numerous factors including patient preference, clinician expertise and experience, type of treatment facility, licencing, and available resources. ${ }^{5}$ Goal of detoxification is the achievement of a drugfree state while minimising withdrawal symptoms.

Opioid detoxification paradigms are frequently categorized according to their duration: longterm (typically 180 days), short-term (up to 30 days), rapid (typically 3-10 days), and ultrarapid (1-2 days), ${ }^{5,6}$ Most common detoxification protocols are the long-term (typically 180 days) and short-term (up to 30 days) paradigms involving use of methadone and buprenorphine. Rapid detoxification involves use of an opioid antagonist like naltrexone and naloxone, partial 
agonists like buprenorphine, in combination with other medications such as clonidine and benzodiazepines, nonsteroidal antiinflammatory agents. 5,6

Clonidine has been shown to supress many of the autonomic signs and symptoms of opioid withdrawal. But, it does not supress body-ache, joint pain and the subjective discomfort of withdrawal and probably for that reason is not well accepted by most patients $5,7,8$

In our centre, while receiving inpatient detoxification treatment for Opioid Dependence, once a patient was prescribed roxithromycin for his associated skin infection for few days. Following clinical improvement of skin lesion, roxithromycin was withdrawn. The very next day patient complained of heightened withdrawal symptoms, especially, diffuse bodyache and joint pain. Patient himself hinted that he was doing well till roxithromycin was prescribed to him and requested for restarting the drug. These symptoms, indeed, relieved again following reintroduction of same antibiotic! Above serendipitous finding led us to put forward a hypothesis that "Roxithromycin helps in reducing certain opioid withdrawal symptoms like body-ache and joint pain", for which no other treatment is currently available except opioid substitution treatment (OST). We decided to give a trial of roxithromycin on other opioid dependent patients who would be getting admitted in our center for opioid detoxification.

\section{MATERIAL AND METHOD}

It was a clinical study spanning over a period of two years. The study set up was the deaddiction centre of the Universal College of Medical Sciences (UCMS), at Bhairahawa, Nepal. Ethical approval for the study was taken from the institute's 'Ethics Approval Committee' prior to starting the study. Individual patient's consent was taken before including their clinical data in the present study. Inclusion criteria were all voluntary inpatients within the age range of 15-45 years with a DSM-IV diagnosis of opioid abuse/dependence. ${ }^{9}$ Exclusion criteria were the history of opioid abuse secondary to functional/organic mental illness and mental retardation.

All the cases that were admitted during the stated period, with a diagnosis of opioid abuse/dependence and/or polysubstance abuse with predominantly opioid dependence were included for initial assessment. Patients were reinterviewed and reassessed in the ward, with history clarified from the patients as well as patients' guardians (wherever present), and the diagnoses were confirmed as per DSM-IV. The DSM-IV questionnaire was used to note down the pattern of drug abuse during the last five years (lifetime diagnosis) and during the last one month (current diagnosis). These patients received standard detoxification protocol that was available at our centre during that period. These include a combination of clonidine, NSAIDs, substitution in the form of tramadol hydrochloride, benzodiazepine for sedation, and at times injection buprenorphine. Antibiotics, vitamin supplementations, antidepressant drug, etc. were given as and when needed basis.

All these subjects were administered a semistructured questionnaire to collect information on the demographic data, duration of opioid drugs (mostly brown sugar) being used and types of opiates used, onset and duration of injection used, if any. A total of 76 opioid abusers were included in the original study. Four patients were excluded for the present assessment, as these 4 patients did not stay even for a day as inpatient, for an effective treatment process to start. Thus, a total of 72 patients have been included for the present study. These patients were followed up regularly in the ward till they get discharged and during subsequent follow-up assessments, if any. Sixteen patients received standard detoxification protocol that was available to us during the period of study. Following above serendipitous discovery, which we mentioned in the introduction, we decided to give a trial of roxithromycin on other opioid dependent patients, who would be getting admitted in our centre for opioid detoxification, during the stipulated study period. For this purpose, we presented a separate proposal for a drug trail before our institute's ethical committee. An ethical clearance was obtained to give a trial of tab roxithromycin. Tab roxithromycin was given in $150 \mathrm{mg}$ twice daily dosage for a period of maximum 10 days or till the patient got discharged, whichever was earlier. This trial was stopped once the original study was stopped. We treated 56 patients with tab roxithromycin, in addition to standard drugs 
that we used in earlier patients. It was noted that there was indeed significant relief in withdrawal symptoms even in patients with no overt infective process.

Simple descriptive statistics such as frequency and percentages, chi-square test, and t-test were used to analyse the data thus available. For carrying out detailed statistical analyses, a computer-assisted statistical package was sought.

\section{RESULT}

We see in the table (Table 1) that the two clinical groups did not differ in most parameters before the start of detoxification treatment.

Average amount of Opioid (Brown sugar) abuse per day (in mg) may appear to be a very high amount. One pack of brown sugar powder usually contains $100 \mathrm{mg}$ of powder. Five to 10 packet of brown sugar abuse per day was very common pattern of abuse in this part of the country!

Though we frequently noted subjective relief of opioid withdrawal symptoms in our patients who received Roxithromycin, we did not use any scale to record this subjective relief vis-a-vis the groups who did not receive macrolide antibiotic.

So, for objective assessment of relief of symptoms, in a retrospective scenario, we thought the best assessment available to us would be the duration of voluntary inpatient stay and the pattern of follow-up of these patients after their discharge.

Below table (Table 1) shows that on both these two parameters roxithromycin recipient group scored significantly higher than their nonrecipient counterpart.

Table 1: Comparison of two clinical groups prior and after treatment

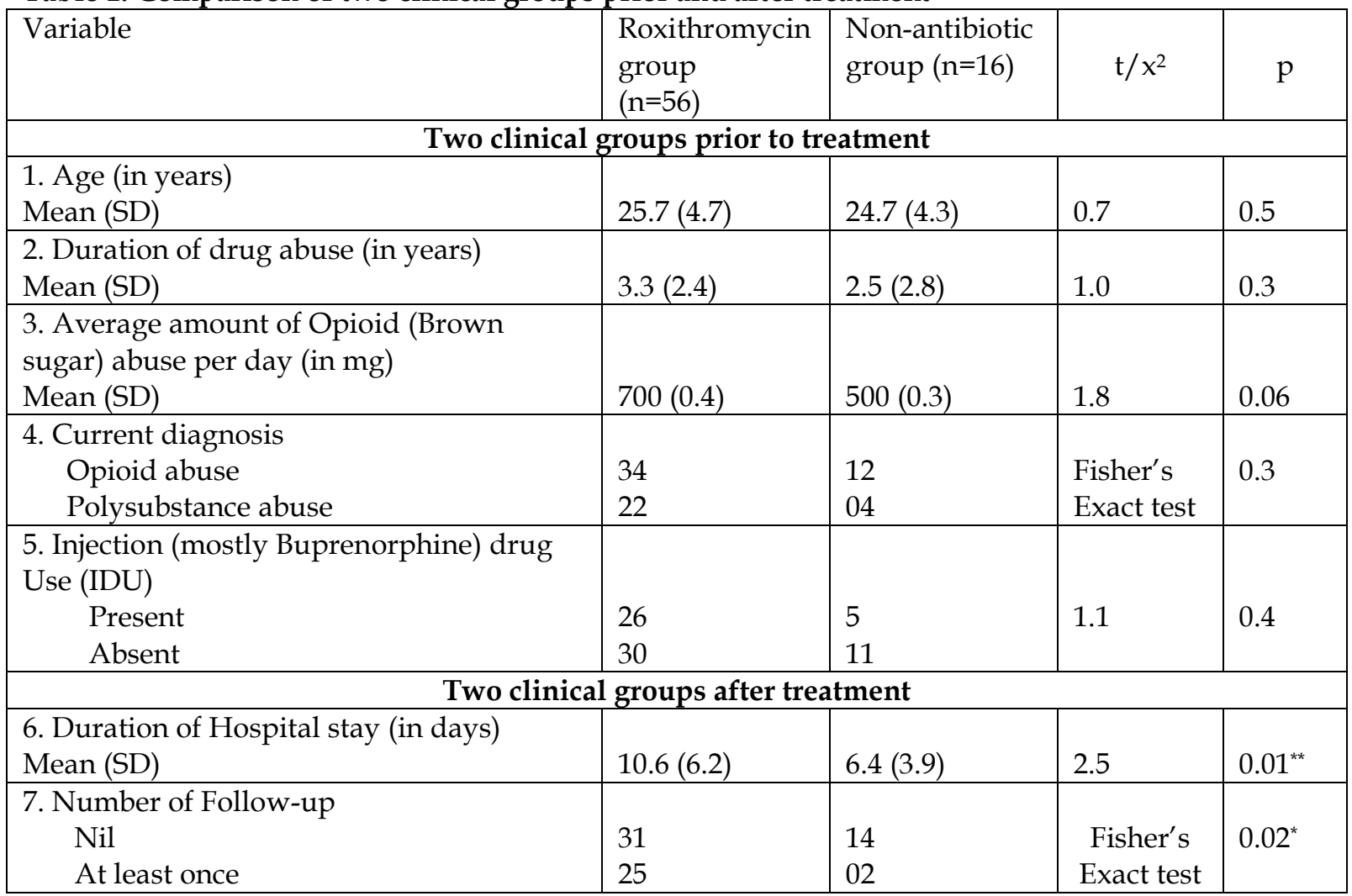

* Significant at 0.01 level; $\quad$ ** Significant at 0.05 level 


\section{DISCUSSION:}

Initial purpose of the present study was to note the demographic and clinical correlates of opioid dependent patients, who were admitted and received treatment for their withdrawal symptoms in our centre. We treated initial 16 patients with the standard treatment protocol that was available to us during that period. It was following the serendipitous discovery we decided to give a trial of Roxithromycin for the rest of our patient population during the study period. Thus, we gave erythromycin tab at the standard adult dose of $150 \mathrm{mg}$ twice a day to the rest 56 patients, in addition to the standard treatment protocol that was given to earlier 16 patients.

It was apparent that Roxithromycin had some clinically significant effect in reducing opioid withdrawal symptoms. How roxithromycin acted in this scenario? Does the effect it produced in reducing withdrawal symptoms was of clinically significant proportion? Was it really a serendipitous discovery, or just an incidental finding, with no significant addition in the treatment armamentarium of opioid detoxification?

There are no direct answers available to these questions even today. The fact that we were not in a position to explain the phenomena observed, prompted us not to report this finding earlier, even though the study was done more than a decade ago and there were already few publications available on the findings from this study. ${ }^{10}$,

However, it was following a chance discussion with our corresponding author $(\mathrm{JH})$ some time back, prompted us to relook into available literature on roxithromycin. Easy availability of literature in net also facilitated the new learning which was hitherto not possible a decade back in a small peripheral medical college in Nepal! We try to provide possible explanation to the observed phenomena as follows:

Roxithromycin basically acts as a bacteriostatic drugs. ${ }^{11}$ Macrolide antibiotics constitute a group of 12 to 16 membered lactone rings substituted with one or more sugar residues. Erythromycin, the first macrolide described, was first isolated in 1952. Roxithromycin is a semi-synthetic macrolide developed to overcome the limitations of erythromycin like gastric acid lability, low oral bioavailability, poor tissue penetration and short half-life. Macrolides exert their antibiotic effect by binding irreversibly to the $50 \mathrm{~S}$ subunit of bacterial ribosomes. This action is mainly bacteriostatic.

Some overt and covert infectious process likely to be there in our patients, who were chronically exposed to opioid drugs of abuse. More vulnerable subgroups were injection drug abusers (IDUs) with history of superficial and deep vein thrombosis, HIV and AIDS and associated chronic secondary infectious processes. Chronic drug abuse also leads to a state of chronic malnutrition and decreased immunity exposing the individual vulnerable to new infectious processes. In such scenario roxithromycin possibly acted an antimicrobial agent leading to a subjective feeling of wellbeing and objective assessment of clinical improvement.

A short note on the drug of abuse: Heroin (Diacetylmorphine) is a fine white powder with a bitter taste in its purest form. It contains up to 85-95\% diacetylmorphine. Non-pure heroin varies in chemical and physical appearance. It contains additives and adulterants, which influence its appearance. Additives make heroin appear as grey, pink/beige, brown, or black granular lumps or black sticky material (black tar). Heroin used in India and Nepal is mostly a brown coarse powder (Brown Sugar) with poor water solubility. Heroin is rapidly hydrolysed in the body to two products-namely, 6monoacetylmorphine (MAM) and morphine. So, pharmacologic effects of brown sugar (heroin), which was being abused by our patients, were dependent largely upon its conversion to MAM and morphine.

Reduced withdrawal intensity: a result of pharmacokinetic (PK) interaction? The potential PK influence of roxithromycin upon other co-administered agents might have influenced outcomes in our study. A reduced withdrawal intensity may have resulted from the demonstrated pharmacokinetic interaction between roxithromycin and opioid of abuse (via P450 enzyme pathways) with a consequent slower elimination of the opioid medication and a prolonged tapering-like effect. Roxithromycin, like other macrolides are, to varying degrees, 
CYP3A4 metabolic inhibitors, and thus it may have influenced enhancing the opioid level in the body.

Opioids pass through two phase pharmacokinetic reactions in the body. Phase I reactions include hydrolysis and oxidation. Most oxidative metabolism is catalysed by cytochrome (CY) P450 enzymes. Phase II reactions make substances more hydrophilic by conjugation to water-loving substances, such as glucuronic acid (Glucuronidation). Phase II reaction is catalysed by uridine di-phosphate glucuronosyltransferase (UGT), the enzyme primarily responsible for metabolizing morphine. Opioids metabolized through Phase II reactions have a much lower potential for drug-drug interactions. Thus morphine is less prone to drug-drug interactions than those opioids eliminated through CYP450 pathways. ${ }^{12}$ Since, in our patients brown sugar (heroin) was the substance of abuse, it was unlikely that Roxithromycin exerted any pharmacokinetic interaction in a clinical scenario like this because heroin does not get metabolised through CYP450 pathways, where roxithromycin exerts its inhibitory action.

Role of roxithromycin as an anti-inflammatory, immunomodulatory drug is known since early 1960s. ${ }^{11,13}$ It was again a chance discovery that erythromycin treatment radically improved the clinical outcome of a patient with diffuse panbronchiolitis in 1960s. Diffuse panbronchiolitis is a progressive inflammatory disorder of lung airways found almost exclusively in Japan. The mechanism of action is thought to be due to immune modifying effects rather than direct antimicrobial activity of erythromycin. This finding rekindled interest in the use of macrolides as a potential treatment in other inflammatory airway disorders.

Macrolides, including roxithromycin, have been shown to have diverse biological activities and an ability to modulate inflammation and immunity in eukaryotes without affecting homeostatic immunity. These properties have led to their long-term use in treating neutrophildominated inflammation in diffuse panbronchiolitis. The anti-inflammatory properties of macrolides form the basis for their experimental use in bronchial asthma and a variety of chronic airway inflammatory and infective conditions. ${ }^{14-16}$ Their spectrum of action extends to the regulation of leukocyte function and production of inflammatory mediators, control of mucus hypersecretion, and resolution of inflammation and modulation of host defence mechanisms ${ }^{16-18}$

Does Macrolide antibiotics and NSAIDS have some synergistic anti-inflammatory action in a clinical situation like this? There is no direct evidence available for or against this statement. We have some supportive documents from animal studies. In an animal study, macrolides were shown to have a tendency towards analgesic potential, though statistically less significant than the conventional analgesics like Ibuprofen. ${ }^{19}$ Ibuprofen is a known inhibitor of Cyclo-oxygenase (COX-2), and by inhibiting COX, it reduces the production of prostaglandins (PGs), thereby causing vasodilation. ${ }^{20,21}$ On the other hand, macrolides inhibit the endothelial injury caused by oxygenderived free radicals, and thus prevent an increase in vascular permeability. Thus, though macrolides does not have established analgesic property, both the drug groups affect hemodynamic component of inflammation, albeit through different routes. ${ }^{19}$

Opioid receptors and their role in the body: $A$ brief review. ${ }^{22}$ The three most important opioid receptors in the brain are $\mu$ - opioid receptor (MOR), $\delta$ - opioid receptor (DOR), K- opioid receptor (KOR). MOR is mostly involved in nociception (pain sensation). MOR, DOR and KOR are G-protein coupled, and activate inhibitory G-proteins. These receptors form homo- and heterodimeric complexes, signal to kinase cascades, and scaffold a variety of proteins. ${ }^{23}$

Opioid drugs act on endogenous opioid neurotransmitter pathway, but with a much greater effect than the natural transmitter. The profound ability of opiates such as heroin to produce addiction is because these drugs highjack the natural transmitter system leaving normal levels of stimulation seeming tame by comparison. ${ }^{24}$

Acute opioid administration lead to the inhibition of adenylate cyclase with reduced 
conversion of ATP to CAMP, resulting in reduced firing at noradrenergic neurones located on the locus coeruleus. Chronic opioid administration leads to compensatory upregulation of cAMP, returning to levels towards baseline. It is important to note that the downregulation of opioid receptors following chronic agonist exposure induces tolerance, as reported in in-vitro studies. ${ }^{25,26}$

On cessation of opioid abuse (or following the opioid receptor antagonism), withdrawal ensues, characterized by a massive surge of unopposed noradrenergic activity (termed the 'noradrenergic storm') from the locus coeruleus. ${ }^{27}$ This noradrenergic hyperactivity is thought to underlie many symptoms of opioid withdrawal, and explains some of the efficacy of the presynaptic a-2 agonists like clonidine in the treatment of acute opioid withdrawal. ${ }^{27}$

What is the possible mode of action linking action of macrolide antibiotics with that of relief of symptoms of opioid withdrawal at cellular level?

The noradrenergic storm during opioid detoxification accompanies oxidative burst within Polymorphonuclear leucocytes (PMNs) and release of pro-inflammatory cytokines like PGF1a, NO-2, and TNF- $\alpha$, and number of Interleukins (IL-1, IL-2, IL-3, IL-4, and IL-6). Proposed mechanisms for the anti-inflammatory activity of macrolides like roxithromycin include accumulation of macrolides and subsequent impairment of this oxidative burst within Polymorphonuclear leucocytes (PMNs) and the resultant decreased production of proinflammatory cytokines like PGF1a, NO-2, TNF$\alpha$, and Interleukins. ${ }^{28,29}$ Of these IL-1, IL-4 and TNF- $\alpha$ have been specifically documented to affect the levels of opioid receptors and pathogenesis of the opioid withdrawal syndrome. Both of these mechanisms are not dependent on direct inhibition of COX-2 as usually done by standard NSAIDS. ${ }^{28,29}$

IL-1 is one of the primary mediators of inflammation. Interleukin-1beta (IL-1 $\beta$ ) is a proinflammatory cytokine that is produced in the central nervous system during inflammatory conditions. It has been shown that IL-1 $\beta$ expression is altered in the rat brain during a morphine tolerant state, indicating that this cytokine may serve as a convergent point between the immune challenge and opiate mediated biological pathways. It appears that IL-1 $\beta$ up-regulates opioid receptors in human astrocytes in both untreated and morphinedesensitized states. Results by Byrne LS et al (2012) show the pro-inflammatory cytokine, IL$1 \beta$, affect opiate-dependent pathways by upregulating the expression of the MOR ( $\mu$ opioid receptor) in both untreated and morphinedesensitized U87 MG human astrocytoma cells. ${ }^{30}$ In their study from 1998, Vidal et al have shown a positive link between expression of MOR in the endothelial cells and the levels of IL$1 .{ }^{31}$

A mechanism for desensitization of opioid receptors (ORs) is the decoupling ("jamming") of the second messenger system associated with the GPCRs (G-protein-coupled receptors) which in many cases is cAMP. This "jamming" is one of the most recently discovered mechanisms behind the downregulation of MOR and OLR-1 (Opioid Like Receptor-1). ${ }^{32}$ Macrolides, including roxithromycin, are known to increase the levels of cAMP thereby possibly resensitizing the OR to the endogenous opioids. Opioid agonists have been found to induce AP-1 (activator protein 1) and NF-KB (nuclear factor kappa-light-chain-enhancer of activated $\mathrm{B}$ cells) transcription factor activity responsible for the increased transcription of pro inflammatory cytokines, possibly leading to increased sensitivity to pain along with joint and body pain. ${ }^{33}$ Macrolides including roxithromycin inhibits this AP-1 and NF-KB transcription factor activity. ${ }^{34}$ This function, also, possibly explains the relief in clinical symptoms of body ache and joint pain after addition of macrolides, during opioid detoxification treatment.

Summary: We observed an interesting clinical phenomenon and the basis of this observation was a serendipitous discovery of the use of roxithromycin by a patient while undergoing detoxification as an inpatient in our deaddiction centre. Though the observation was noted more than a decade back, we decided to repot it now only, after we gathered, as we believe, adequate proof in favour of using roxithromycin in the opioid detoxification protocol.

Summarising the possible mode of actions of roxithromycin in opioid withdrawal syndrome are: firstly, antimicrobial action of roxithromycin 
was probably helped in certain sub-group of our patients in relieving their clinical withdrawal symptoms. Secondly, immunomodulatory and anti-inflammatory properties of roxithromycin, probably, and more importantly, responsible for sustained symptomatic relief from withdrawal symptoms in most of our patients. Thirdly, roxithromycin also, probably, has some synergistic action with clonidine and NSAIDS in such a clinical scenario. Finally, a reduced withdrawal intensity may also result from the demonstrated pharmacokinetic interaction between roxithromycin and opioid of abuse (via P450 enzyme pathways) with a consequent slower elimination of the opioid medication and a prolonged tapering-like effect.

To the best of our current knowledge there is no reported use of roxithromycin in 'Opioid detoxification Programme' till date. The study needs to be replicated in robust double blind randomised controlled trials, to validate the use of roxithromycin in the opioid detoxification programme in future.

Limitations: Following are the number of limitations, we envisage, leading to cautious interpretation and acceptance of the findings:

1. There was no random assignment of patients to roxithromycin and non-roxithromycin group. Thus the treatment was non-blind and the interpretation is difficult to generalise. There is no comparative placebo group to match roxithromycin users, once roxithromycin was introduced in the treatment regimen.

Since it was an ongoing naturalistic study, with no prior thought of a drug study, a methodically sound drug study could not be done. However, we think, that does not discount the significance of the findings of the present study.

2. Since it was a retrospective assessment we utilized the duration of voluntary inpatient stay and the number of follow-up visits patients had after their discharge as a way of gauging the influence of the roxithromycin. The outcomes chosen do not seem apt to evaluate the validity of the treatment. We accept that the criteria we have chosen as a proxy for "relief of symptoms" is poor.

In the absence of any other supporting measures of withdrawal, duration of voluntary inpatient stay and self-review pattern appeared to be fairly good indicators treatment completion. We accept that this is a weak assessment of the effect of roxithromycin but it is what was available to us and used.

3. It is likely that those prescribed antibiotics have more symptoms than those who do not, which accounts for the longer stay and higher number of follow-up visits.

This is unlikely be the cause, since the two groups did not differ on various demographic and clinical profiles prior to start of treatment.

4. The suggested off-label use of medications to treat opioid withdrawal raises safety issues in terms of risk of potential antibiotic side effects and the development of antibiotic resistance in the treated patients.

We have used roxithromycin only for a maximum period of 10 days or till the patient got discharged, whichever was the shorter period. Literature is available where roxithromycin and other macrolides have been used for months in other chronic inflammatory states. So, apprehension of developing antibiotic resistant state was very unlikely.

\section{ACKNOWLEDGEMENT: None}

\section{CONFLICT OF INTEREST: None}

\section{REFERENCES:}

1. Soyka M, Rothenhausler HB. Pharmacotherapies of opioid detoxification-an evaluation of different pharmacotherapeutical strategies. Alcohol Treat $Q$. 1999; 17(3):47-53.

2. Stine SM, Kosten TR. Use of drug combinations in treatment of opioid withdrawal. J Clin Psychopharmacol 1992; 12(3):203-209.

3. Pani PP, Maremmani I, Pirastu $R$, et al. Buprenorphine: A controlled clinical trial in the treatment of opioid dependence. Drug Alcohol Depend. 2000; 60(1):39-50.

4. Schneider $U$, Paetzold $W$, Eronat $V$, et al. Buprenorphine and carbamazepine as a treatment for detoxification of opiate addicts with multiple drug misuse: A pilot study. Addict Biol 2000; 5(1):65-69.

5. Woody GE, Fudala PJ. Substance Abuse: Opioid use disorders. In: Psychiatry $3^{\text {rd }}$ ed. Eds. Tasman A, Kay J, Lieberman JA et al. Wiley-Blackwell, West Sussex, England 2008; 63:1170-85. 
6. O'Connor PG, Kosten TR. Rapid and ultrarapid opioid detoxification techniques. I Am Med Assoc. 1998; 279(3):229-234.

7. Neil MJ. Clonidine: clinical pharmacology and therapeutic use in pain management. Curr Clin Pharmacol. 2011; 6(4):280-7.

8. Stahl SM. Clonidine. In: Stahl's essential psychopharmacology: prescriber's guide, $5^{\text {th }}$ ed. Cambridge: Cambridge University Press, 2014; 14347.

9. American Psychiatric Association (APA). Diagnostic and Statistical Manual of Mental Disorders, $4^{\text {th }}$ ed. (DSM-IV), Washington DC: APA 1994.

10. Aich TK, Dhungana $M$, Khanal R. Pattern of buprenorphine abuse amongst opioid abusers in Nepal. Indian J Psychiatry 2010; 52(3):250-253.

11. Gudin J. Opioid Therapies and Cytochrome P450 Interactions. J Pain Symptom Manage 2012; 44(6S):414.

12. Kanoh S, Rubin BK. Mechanisms of Action and Clinical Application of Macrolides as Immunomodulatory Medications. Clin Microbiol Rev Downloaded http://cmr.asm.org/content/23/3/590.full

13. Crosbie PAJ, Woodhead MA. Long-term macrolide therapy in chronic inflammatory airway diseases. Eur Respir J 2009; 33: 171-181

14. Itkin IH, Menzel ML. The use of macrolide antibiotic substances in the treatment of asthma. J Allergy 1970; 45:146-162.

15. Konno SI, Asano K, Kurokawa M, et al. Antiasthmatic Activity of a Macrolide Antibiotic, Roxithromycin: Analysis of Possible Mechanisms in vitro and in vivo. Int Arch Allergy Immunol 1994;105:308-316.

16. Cazzola M, Matera MG, Blasi F. Macrolide and occult infection in asthma. Curr Opin Pulm Med 2004 Jan;10(1):7-14.

17. Tamaoki J, Kadota J, Takizawa H. Clinical implications of the immunomodulatory effects of macrolides. Am J Med 2004;117: Suppl. 9A 5S-11S.

18. Spagnolo P, Fabbri LM, Bush A. Long-term macrolide treatment for chronic respiratory disease. Eur Respir J 2013; 42: 239-251

19. Khobragade AA, Patel SB, Pophale RR, et al. Analgesic and Anti-inflammatory Activity of Roxithromycin and Erythromycin, Alone and in Combination with Ibuprofen: An Animal Study. IOSR J Pharmacy 2012; 1(1):15-21.

20. Burke A, Smyth E, FitzGerald GA. AnalgesicAntipyretic Agents; Pharmacotherapy of Gout. In: Laurence LB, editor. Goodman $\mathcal{E}$ Gilman's The Pharmacological Basis of Therapeutics 11th ed. New York: McGraw Hill; 2006; 698.

21. Vinay K, Abul KA, Nelson F. Acute and Chronic inflammation. In: Robbins and Cotran editors. Pathologic Basis of Disease, 7th ed. 2005; New Delhi: Elsevier: 47-86.

22. Waldhoer M, Bartlett SE, Jennifer L. et al. Opioid receptors. Annu Rev Biochem 2004; 73:953 -990.
23. Mayet S, Winstock AR, Strang J. Opioids: heroin methadone and buprenorphine. In: Gelder MG Andreasen NC, Lopez-Ibor Jr JJ et al (eds). New Oxford textbook of psychiatry, $2^{\text {nd }}$ ed. United Kingdom: Oxford University Press, 2009; 473-82.

24. Nutt JD, Law DF. Pharmacological and psychological aspects of drugs abuse. In: Gelder MG, Andreasen NC, Lopez-Ibor Jr JJ et al (eds). New Oxford textbook of Psychiatry, $2^{\text {nd }}$ ed. United Kingdom: Oxford University Press, 2009; 427-32.

25. Harrison RS, Ruiz-Gómez G, Hill TA, et al. Novel Helix-Constrained nociceptin derivatives are potent agonists and antagonists of ERK phosphorylation and thermal analgesia in mice. J Med Chem 2010; 53:840008 .

26. Dang VC, Christie MJ. Mechanisms of rapid opioid receptor desensitization, resensitization and tolerance in brain neurons. Br J Pharmacol 2011.

27. Simonato $M$. The neurochemistry of morphine addiction in the neocortex. Trends Pharmacol Sci. 1996; 17:410-15.

28. Labro MT, Benna JE, Babin-Chevaye C. Comparison of the in-vitro effect of several macrolides on the oxidative burst of human neutrophils. J Antimicrob Chemother 1989; 24: 561-72.

29. Martina B, Zeljko K, Vesna $M$, et al. Cellular Uptake and Efflux of Azithromycin, Erythromycin, Clarithromycin, Telithromycin, and Cethromycin. Antimicrob Agents chemother 2005 June; 2372-77.

30. Byrne LS, Peng J, Sarkar S, et al. Interleukin-1 beta induced up-regulation of opioid receptors in the untreated and morphine-desensitized U87 MG human astrocytoma cells. J Neuroinflammation 2012;9:252.

31. Vidal EL, Patel NA, Wu G, et al. Interleukin-1 induces the expression of $\mu$ opioid receptors in endothelial cells. Immunopharmacol 1998; 38(3):261-266.

32. Aoshiba K, Nagai A, Konno K. Erythromycin shortens neutrophil survival by accelerating apoptosis. Antimicrob Agents Chemother 1995; 39(4):872-877.

33. Kikuchi T, Hagiwara K, Honda Y, et al. Clarithromycin suppresses lipopolysaccharide-induced interleukin-8 production by human monocytes through $A P-1$ and NF- $\kappa B$ transcription factors. J Antimicrob Chemother 2002; 49 (5): 745-755.

34. Choi Y, Koh SJ, Lee HS, et al. Roxithromycin inhibits nuclear factor kappaB signalling and endoplasmic reticulum stress in intestinal epithelial cells and ameliorates experimental colitis in mice. Exp Biol Med 2015; 240(12):1664-1671. 